\title{
INTEGRAĈ̣̃O RIS/PACS NO HOSPITAL DAS CLÍNICAS DE RIBEIRÃO PRETO: UMA SOLUC̣ÃO BASEADA EM "WEB"*
}

\author{
Paulo Mazzoncini de Azevedo-Marques ${ }^{1}$, Edilson Carlos Caritá ${ }^{2}$, Alexander Antonio Benedicto ${ }^{3}$, \\ Pablo Rodrigo Sanches ${ }^{4}$
}

Resumo OBJETIVO: Integração dos Sistemas de Informação em Radiologia (RIS - “Radiology Information System") e de Arquivamento e Comunicação de Imagens (PACS - "Picture Archiving and Communication System") no Serviço de Radiodiagnóstico do Hospital das Clínicas da Faculdade de Medicina de Ribeirão Preto da Universidade de São Paulo, para possibilitar a consulta remota de laudos e imagens associadas. MATERIAIS E MÉTODOS: A integração RIS/PACS implementada é feita em tempo real, no momento da consulta, utilizando tecnologias "web" e técnicas de programação para "intranet/internet". RESULTADOS: A aplicação "web" permite a consulta pela "intranet" do hospital dos laudos de exames e imagens associadas através de nome, sobrenome, número de registro hospitalar dos pacientes ou por modalidade, dentro de um determinado período. $\mathrm{O}$ visualizador possibilita que o usuário navegue pelas imagens, podendo realizar algumas funções básicas como "zoom", controle de brilho e contraste e visualização de imagens lado a lado. CONCLUSÃO: A integração RIS/PACS diminui o risco de inconsistências, através da redução do número de interfaces entre bases de dados com grande redundância de informação, proporcionando um ambiente de trabalho rápido e seguro para consulta de laudos radiológicos e visualização de imagens associadas.

Unitermos: PACS; RIS; Radiologia sem filme; Tecnologia "web".

Abstract RIS/PACS integration at "Hospital das Clínicas de Ribeirão Preto": a web based solution.

OBJECTIVE: Integration of the Radiology Information System (RIS) and the Picture Archiving and Communication System (PACS) of the Radiodiagnosis Service of “Hospital das Clínicas de Ribeirão Preto da Universidade de São Paulo", Brazil, to allow remote access to reports and associated images. MATERIALS AND METHODS: RIS/PACS integration occurs in real time at the moment of consultation, using web technologies and programming techniques for intranet/internet. RESULTS: This web application allows reports and associated images viewing through the medical center intranet. Search is based on the patients' first name, last name, hospital number identification and exam modality, within a time period. The viewer allows image navigation and presents some basic functions such as zoom, brightness/contrast control and side-by-side image visualization. CONCLUSION: RIS/PACS integration minimizes the risk of inconsistencies, through the reduction of the number of interfaces among data bases with great information redundancy, providing a fast and safe work environment for radiology reports, consultation and visualization of associated images.

Key words: PACS; RIS; Filmless radiology; Web technology.

\footnotetext{
* Trabalho realizado junto ao Serviço de Radiodiagnóstico do Hospital das Clínicas da Faculdade de Medicina de Ribeirão Preto da Universidade de São Paulo (FMRP-USP), Ribeirão Preto, SP Trabalho parcialmente financiado pela Fundação de Amparo à Pesquisa do Estado de São Paulo (Fapesp), Processo no 99/ 10875-5.

1. Engenheiro Eletrônico, Doutor em Física Aplicada, Docente do Centro de Ciências das Imagens e Física Médica (CCIFM) da FMRP-USP.

2. Bacharel em Processamento de Dados, Mestre em Engenharia Elétrica, Aluno de Doutorado junto ao CCIFM/FMRPUSP.

3. Analista de Sistemas, Especialista em Sistemas de Informação, Aluno de Mestrado junto ao CCIFM/FMRP-USP, Analista de Sistemas do Centro de Informações e Análises do Hospital das Clínicas de Ribeirão Preto.

4. Bacharel em Análise de Sistemas, Analista de Sistemas do Centro de Informações e Análises do Hospital das Clínicas de Ribeirão Preto.

Endereço para correspondência: Dr. Paulo Mazzoncini de Azevedo Marques. Rua Ceará, 3005, ap. 22, Jardim Paulistano. Ribeirão Preto, SP, 14090-300. E-mail: pmarques@fmrp. usp.br

Recebido para publicação em 22/9/2003. Aceito, após revisão, em 26/2/2004.
}

\section{INTRODUÇÃO}

Os sistemas de informação para gerenciamento de imagens e informações clínicas surgiram no final da década de 80 , quando os processos de aquisição digital começaram a ser utilizados em larga escala nos hospitais. Naquela época, cada equipamento era considerado um sistema isolado, estando conectado somente à sua estação de trabalho e a uma determinada impressora $^{(\mathbf{1})}$.

O potencial diagnóstico dos primeiros sistemas, em conjunto com o crescimento dos processos de distribuição de informação em formato digital, criou a necessidade de estabelecer um padrão para a obtenção e comunicação de imagens médicas em formato digital. Em resposta a esta necessidade, a National Electrical Manufacturers Association (NEMA) e a Radiology Society of North America (RSNA), em conjunto com uma série de empresas e universidades americanas, criaram o padrão DICOM ("Digital Imaging and Communication in Medicine"). O DICOM foi apresentado ao público pela primeira vez no encontro de 1993 da RSNA ${ }^{(1,2)}$.

A padronização através do DICOM foi fundamental para o desenvolvimento e implantação dos Sistemas de Arquivamento e Comunicação de Imagens (PACS - "Picture Archiving and Communication System"). O PACS vem-se tornando rapidamente a melhor opção tecnológica para as tarefas de transmissão, armazenamento e 
recuperação de imagens médicas, formando em conjunto com os Sistemas de Informação em Radiologia (RIS - "Radiology Information System") e de Informação Hospitalar (HIS - "Hospital Information System") a base para um serviço de radiologia sem filme ("filmless").

Radiologia "filmless" refere-se a um hospital com um ambiente de rede de computadores amplo e integrado, no qual o filme foi completamente, ou em grande parte, substituído por sistemas eletrônicos que adquirem, arquivam, disponibilizam e exibem essas imagens.

A implantação de um serviço de radiologia "filmless" tem como objetivo trazer melhorias na acessibilidade, integração das informações pela vinculação de imagens ao registro eletrônico do paciente, na aplicação de novas técnicas para o desenvolvimento de novas formas de aquisição, exibição e processamento de imagens, e deve também impactar de forma econômica nestes serviços pela redução de gastos com insumos para a radiologia convencional e a eliminação de desperdícios de materiais na repetição de exames. Segundo a literatura especializada, a maioria dos serviços médicos deve fazer a transição para a radiologia "filmless" no período dos próximos dez a vinte anos ${ }^{(\mathbf{3})}$.

Em um ambiente "filmless", a integração RIS/PACS é a base para o bom funcionamento do serviço, prevenindo a inconsistência de informações por meio da integração do conteúdo dos bancos de dados envolvidos no processo. Uma opção para se fazer esta integração é a utilização de tecnologia "web", por ser baseada em padrões internacionais e propiciar uma fácil e rápida distribuição das informações com uma curva de aprendizado muito rápida para o usuário final. Além disso, possui as principais características necessárias para a integração de sistemas baseados em arquiteturas cliente/servidor. Nesta arquitetura, um computador servidor cuida dos processos e operações complexos e a comunicação com o computador cliente é feita através de um protocolo padrão. Além disso, a interface para visualização de dados e imagens pode ser padronizada e incorporada no sistema operacional da máquina cliente, através de um "browser". Desse modo, as máquinas cliente e servi- dor podem operar de forma independente e a transmissão de dados é controlada em nível de sistema operacional, proporcionando maior garantia em termos de eficiência e estabilidade ${ }^{(\mathbf{1})}$.

O Centro de Ciências das Imagens e Física Médica (CCIFM) da Faculdade de Medicina de Ribeirão Preto da Universidade de São Paulo (FMRP-USP) vem trabalhando com o objetivo de propiciar a transição do Serviço de Radiodiagnóstico do Hospital das Clínicas da FMRP-USP para a um ambiente "filmless". Esse processo iniciou-se em 1998, com a proposta de desenvolvimento e implantação de um RIS $^{(4)}$, seguido pela introdução do PACS. A implantação dos sistemas teve início em 1999 e continua em andamento. O RIS está em produção desde agosto de 1999 e a integração RIS/PACS foi feita no final de 2002. Este trabalho descreve o estado atual do projeto "FilmLess-HCFMRP", bem como algumas aplicações que estão sendo desenvolvidas. Serão também apresentados os resultados das nossas primeiras experiências com a utilização dos sistemas implementados.

\section{MATERIAIS E MÉTODOS}

O projeto "FilmLess-HCFMRP" teve como objetivo inicial a criação dos sistemas base para um serviço sem filme, o que, conseqüentemente, implicou a criação do RIS e do PACS-HCRP. Estes sistemas foram desenvolvidos em momentos diversos devido à sua própria complexidade e também à necessidade de um planejamento para atender as prioridades do Serviço de Radiodiagnóstico do Hospital das Clínicas. Isto resultou em um conjunto de sistemas heterogêneos que realizam tarefas específicas que abragem desde o registro do paciente até a geração de suas imagens. Considerando o fato que estas informações, apesar de serem do mesmo paciente, estão dispersas nas bases destes sistemas que formam a base "filmless", há a necessidade de vinculá-las através de uma chave forte o suficiente para garantir a sua paridade referencial. Dessa forma, surgiu a necessidade de implementar um novo sistema para a integração desses dados, possibilitando, assim, a consulta eletrônica de laudos vinculados às suas respectivas imagens.
O RIS-HCRP (Sistema de Informação de Radiologia do Hospital das Clínicas de Ribeirão Preto) foi desenvolvido para funcionar sob uma arquitetura cliente/servidor, com modelo de dados relacional e interface gráfica amigável, proporcionando uma fácil operabilidade para o usuário final. Atualmente, toda a base de informações RIS e HIS está armazenada em um banco de dados Oracle, versão 8.1.7.0.0, e suas interfaces "front-end" implementadas em Delphi 5 (linguagem de programação visual). Os módulos principais do RIS são: módulo de geração de exames, módulo de geração de laudos, módulo de consulta de laudos e módulo de gerenciamento. O acesso a esses módulos é definido pelos direitos concedidos a cada um dos profissionais que devem ser cadastrados no sistema com um nome de usuário e senha. No momento em que o sistema é iniciado, são solicitados o nome de usuário e a senha do profissional. Dessa forma, o sistema permite que sejam acessados somente os módulos que foram previamente concedidos conforme a sua função no fluxo de atividades no serviço. Quando é solicitado um exame para um paciente, é gerada pelo HIS uma etiqueta com código de barras contendo o número de registro do paciente e a clínica solicitante. No momento da realização do exame, o técnico gera o registro eletrônico no módulo de geração de exames através da leitura deste código de barras e em seguida faz a inserção das informações referentes ao tipo de exame, região anatômica e técnica empregada (Figura 1). Ao finalizar a realização do exame, os filmes são encaminhados para a sala de leitura para serem avaliados e laudados. Os laudos são feitos inicialmente pelos médicos residentes e posteriormente conferidos pelos docentes e médicos contratados. Dessa forma, esses laudos recebem inicialmente, no sistema, o status de laudo provisório, não sendo visíveis na rede do HCRP até que o médico revisor faça a revisão desses laudos e os libere como definitivos. Após a revisão, os laudos definitivos já podem ser acessados por qualquer estação do HCRP através de sua "intranet" pelo módulo de consulta, sendo também impressos diretamente no Serviço de Arquivo Médico (SAM), onde são anexados ao prontuário do paciente. Detalhes sobre 
o RIS podem ser encontrados em artigo publicado anteriormente ${ }^{(\mathbf{4})}$.

O PACS-HCRP (Sistema de Arquivamento e Comunicação de Imagens do Hospital das Clínicas de Ribeirão Preto) é composto por um conjunto de "software" e "hardware" formando uma estrutura com vários serviços, como: servidor DICOM, servidor para o armazenamento de imagens DICOM ("storage"), interface de integração com o servidor RIS/HIS e servidor "web" (Figura 2). O servidor DICOM utilizado neste trabalho é o CTN ("central test node"), constituído de vários programas "open-source" desenvolvidos pelo Mallinckrodt Institute of Radiology - Electronic Radiology Laboratory, USA, com o objetivo de demonstrar a conectividade do padrão DICOM. O CTN usa um banco de dados relacional para armazenar suas configurações e os dados referentes às imagens recebidas por ele. Em sua estrutura de configuração há uma tabela denominada "Application Entity”, em que são cadastrados todos os equipamentos que interagem no processo de comunicação das imagens. É importante salientar que o CTN somente suporta comunicações de rede TCP/IP. Além de suas tabelas nas quais estão armazenados os dados com as configurações para a comunicação das imagens, o CTN também possui vários serviços, que são programas que rodam tanto do lado do servidor como do lado cliente, para garantir que a comunicação ocorra de maneira correta. O principal serviço responsável pelo recebimento e armazenamento das imagens DICOM é o "archive_server", o qual recebe as requisições dos equipamentos clientes, armazena os dados das "tags" do arquivo que está recebendo em suas tabelas e grava o arquivo DICOM em diretório. As "tags" são atributos do DICOM onde estão armazenadas as informações referentes ao paciente e o seu exame. Geralmente as informações que são armazenadas nas tabelas do CTN são os dados do paciente, do exame e a localização onde o arquivo DICOM original foi gravado no servidor (Figura 3).

As principais tabelas do CTN são: "patientlevel" - informações do paciente como identificação, nome, data de nascimento, sexo, etc.; "studylevel" - informações referentes aos estudos do exame que

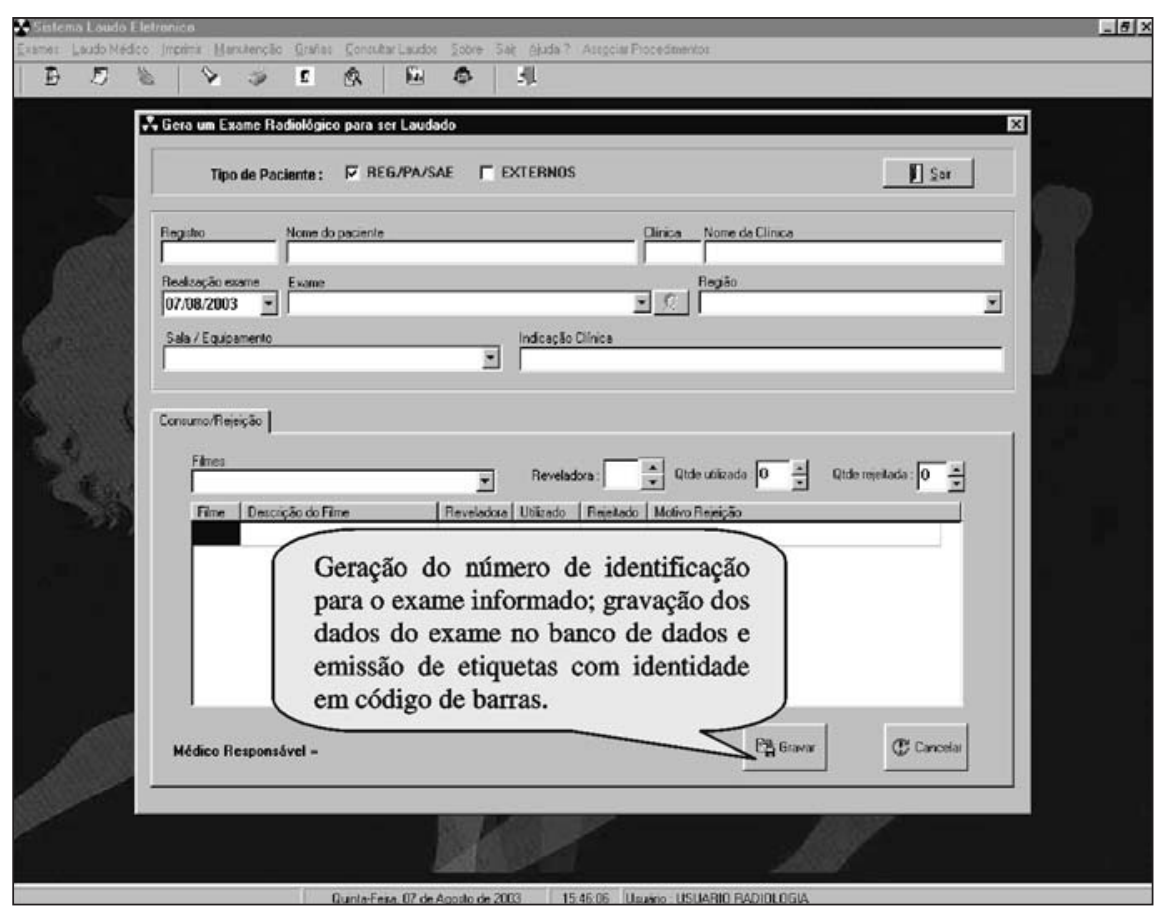

Figura 1. Interface RIS. Informação dos dados do exame e geração do número de identificação (“accession number").

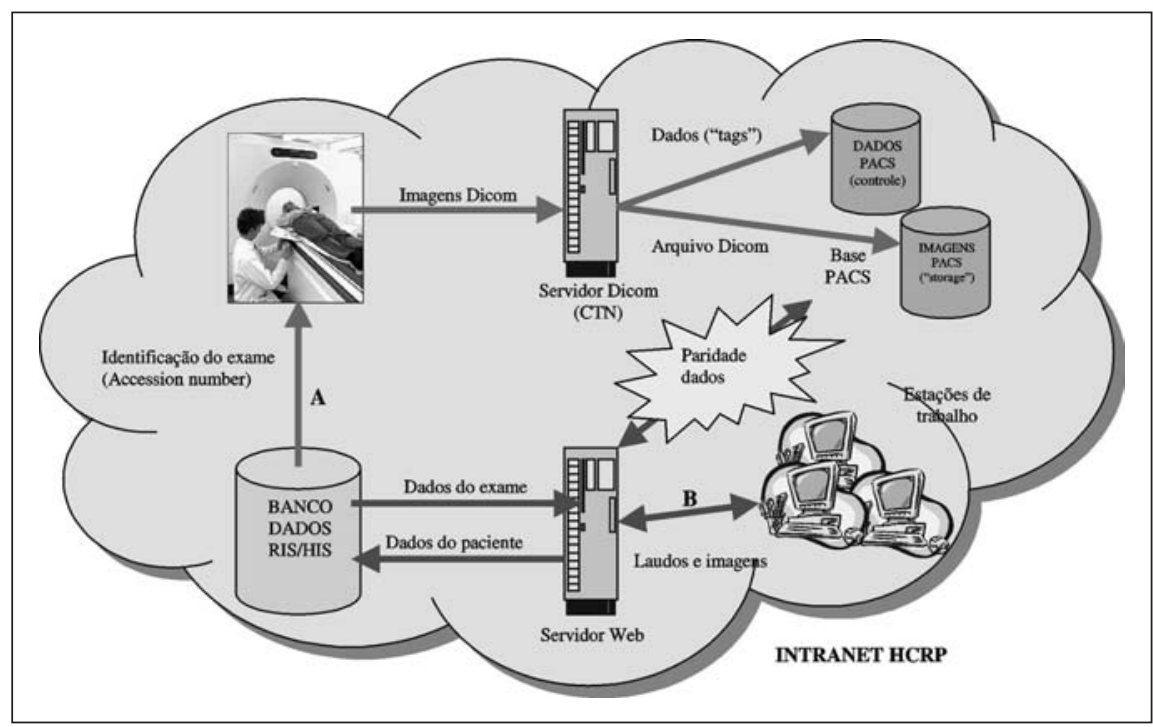

Figura 2. Estrutura PACS-HCRP. Fluxo A: aquisição e armazenamento de imagens DICOM. Fluxo B: recuperação e exibição do laudo vinculado à imagem.

são definidos durante a aquisição das imagens (é nesta tabela que a informação da "tag - accession number" é armazenada através da coluna "accnum"); "serieslevel" - informações sobre as séries do exame; "imagelevel" - informações específicas sobre as imagens; e "instancetable" - tabela que contém o ponteiro indicando onde o arquivo DICOM original foi grava- do no servidor através da coluna "path" (Figura 4).

Deve-se ressaltar que esta estrutura de tabelas é extremamente importante, pois é através dela que se faz a paridade e vinculação das informações dos dados entre as bases RIS/PACS. Considerando o fato de que os arquivos DICOM podem se tornar muito grandes, dependendo do tipo de 


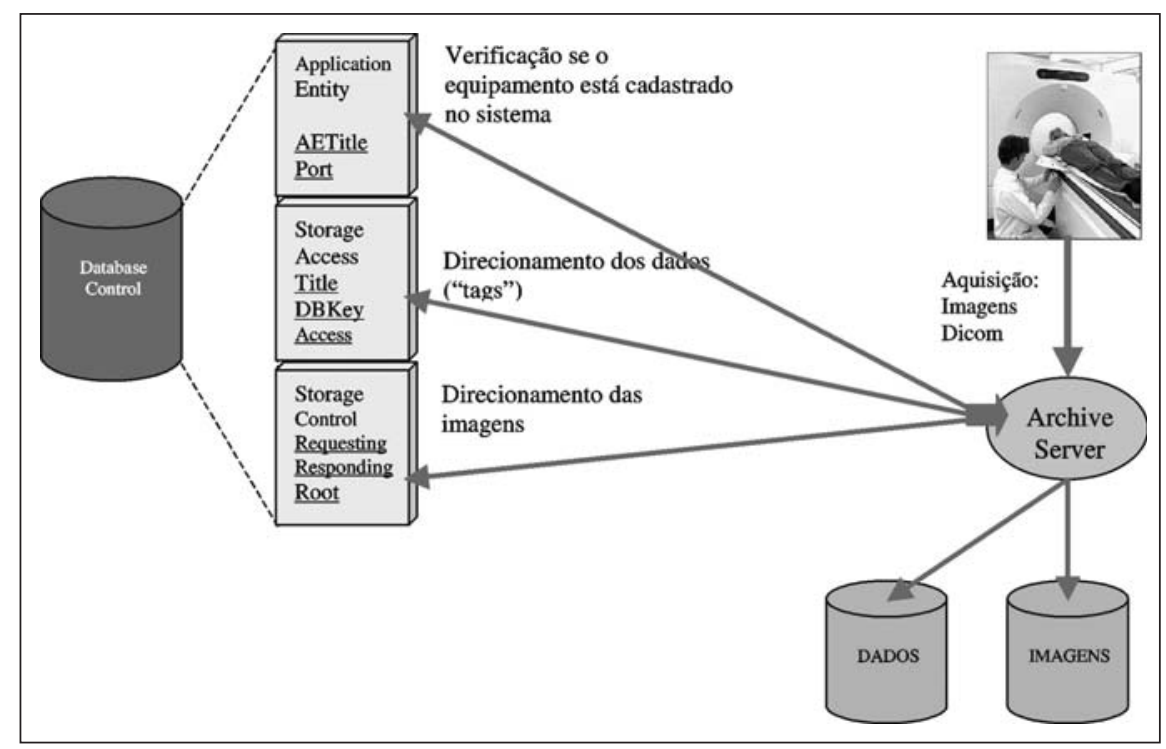

Figura 3. Estrutura simplificada de recebimento e armazenamento de imagens DICOM pelo CTN.

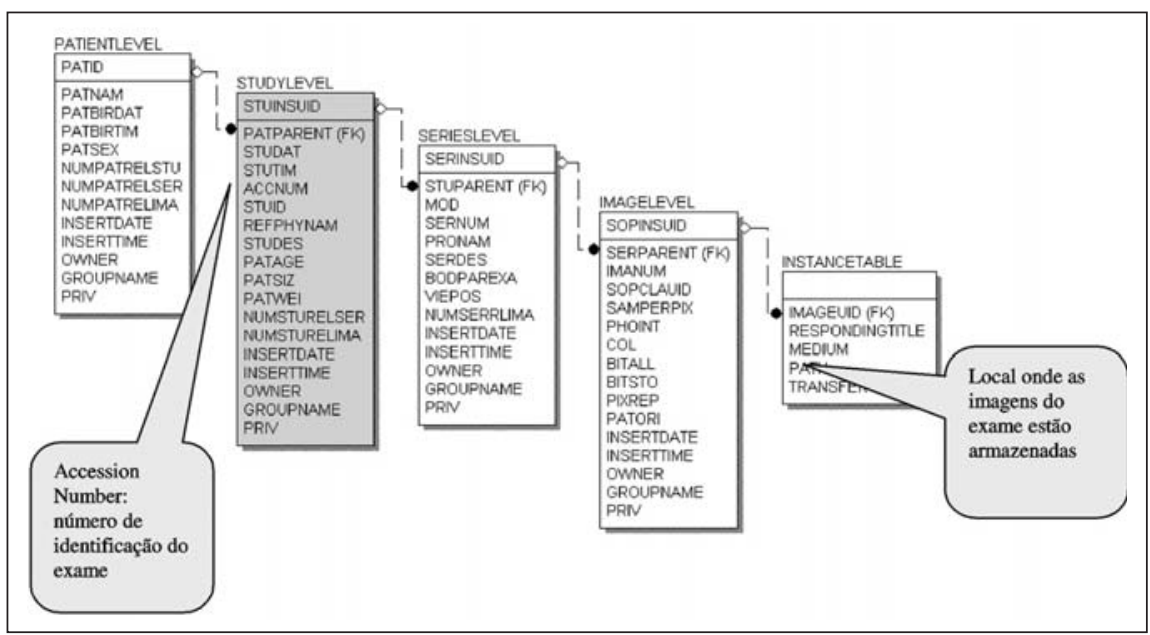

Figura 4. Informações extraídas das "tags" dos arquivos DICOM durante o processo de armazenamento e gravadas nas tabelas do CTN.

exame que ele possui, é necessária grande área para o seu armazenamento no servidor. Para isto, um outro servidor com 200 GB de espaço disponível foi incorporado à estrutura PACS, com a finalidade de armazenar estas imagens ("storage"), possibilitando assim sua consulta "on-line".

O sistema PACS-HCRP é uma continuação do processo de atendimento de pacientes para a realização de exames para diagnóstico por imagem. Depois do exame devidamente gerado no RIS, o técnico digita as informações de identificação do paciente e do exame na interface do equipamento onde serão geradas as imagens (por exemplo: ressonância magnética, ultra-so- face do equipamento que gera o exame, através da seleção do "Aetitle" ("string” de identificação das entidades-equipamentos que fazem parte da rede PACS) do servidor que receberá e armazenará estes arquivos, neste caso o CTN, que foi devidamente configurado e cadastrado no equipamento que está gerando as imagens. Quando o CTN recebe os arquivos DICOM, as informações contidas nas "tags" dos arquivos, como a identificação do paciente e a identificação do exame, são armazenadas nas tabelas de seu banco de dados e o arquivo original é gravado em uma determinada pasta que é criada no servidor de armazenamento ("storage"), sob o número de identificação do exame informado na "tag - accession number". Dessa forma, as informações dos pacientes e seus respectivos exames são compartilhados nas bases dos sistemas RIS/PACS, possibilitando fazer a paridade e consistência dos dados no momento da integração dos laudos com as imagens, garantindo, assim, a confiabilidade da vinculação.

A partir da realização destas etapas preliminares é possível realizar efetivamente a integração do RIS/PACS. Para isto, utiliza-se a tecnologia "web", com acesso e distribuição das informações desses sistemas através de uma aplicação desenvolvida em HTML ("hiper text markup language") e ASP ("active server pages"), rodando sob um servidor "web"-IIS ("internet information server") e com conexão para as duas bases de dados via ADO ("activeX data objects").

A vinculação dos laudos e das imagens é feita em tempo de execução pelo sistema "web" "intranet"-HCRP (Figura 5). Durante o processo de consulta de laudos via "web", uma janela com uma interface amigável é aberta para o médico que deseja visualizar os exames de um determinado paciente, solicitando seu nome de usuário e senha para o acesso. $\mathrm{O}$ sistema oferece ao usuário algumas opções, e dentre estas a consulta de laudo eletrônico. Selecionando esta opção, abre-se uma nova janela em que o usuário pode informar o número de registro de um paciente específico ou solicitar a recuperação de todos os exames de uma determinada modalidade, dentro de um determinado período de tempo. Uma vez selecionado o paciente e o exame que 


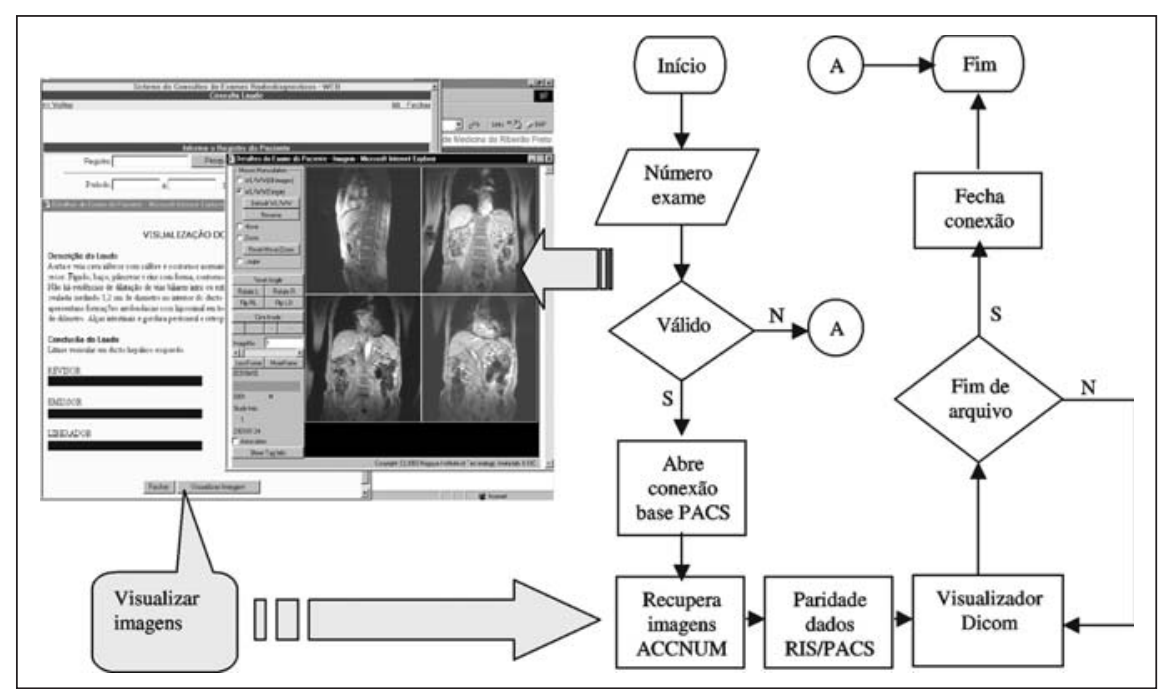

Figura 5. Interface "web" para consulta e fluxograma de acesso, associação e exibição das imagens Dicom com o laudo.

se deseja visualizar, abre-se uma nova janela com o laudo do exame e as informações sobre os profissionais que participaram da sua elaboração. Neste momento, o sistema faz a vinculação com o RIS/PACS e verifica na base de dados do CTN se há imagens "on-line" para esse exame. Caso a resposta seja positiva, exibe-se um botão na parte inferior da janela do laudo indicando que há imagens que podem ser visualizadas. Quando o usuário "clica" neste botão, o sistema cria uma estrutura de "loop" para recuperar todas as imagens referentes ao exame selecionado e abre uma nova janela iniciando um "applet" Java para a visualização das imagens DICOM. Este "applet" Java, denominado "Dicom viewer", é um "software opensource" desenvolvido e disponibilizado pelo Nagoya Institute of Technology, Iwata Laboratory, Japão, com o objetivo de fazer a visualização de imagens DICOM a partir de um "web browser".

\section{RESULTADOS}

O RIS foi inicialmente implantado nos setores de ressonância magnética e tomografia computadorizada do HCFMRP. O que favoreceu essa escolha foi o menor volume de exames realizados nesses setores em comparação com outros - como o de radiologia convencional, por exemplo - e ao fato de seus profissionais técnicos já possuírem familiaridade com o uso de computadores, o que é inerente a essas técnicas de geração de exames. Isto possibilitou o ajuste do sistema sem maiores prejuízos ao andamento dos trabalhos junto ao Serviço de Radiodiagnóstico. Após um período inicial de utilização e ajustes, o sistema foi estendido aos demais setores do Serviço de Radiodiagnóstico. Com isso, todos os exames radiológicos atualmente realizados no HCFMRP estão sendo gerados e laudados dentro do ambiente do RIS, podendo ser consultados via "browser" em qualquer computador conectado à "intranet" do hospital.

O PACS-HCRP, igualmente ao RIS, também foi implantado inicialmente no setor de ressonância magnética pelos mesmos motivos descritos anteriormente e principalmente pelo fato da compatibilidade dos seus equipamentos com o padrão DICOM. O sistema ainda encontra-se em testes, mas já se mostrou eficaz para o seu propósito e possibilitou a implantação de sistemas para laudo eletrônico com o enfoque "filmless" também em outras áreas do hospital, como a Ginecologia e Obstetrícia e a Endoscopia Digestiva.

A aplicação "web" permite a consulta dos laudos de exames através de nome, sobrenome, número de registro hospitalar dos pacientes ou por modalidade, dentro de um determinado período. Quando um paciente é selecionado, abre-se uma nova janela com os exames encontrados conforme os parâmetros solicitados. Dessa forma, o médico pode selecionar o exame que the é de interesse, visualizando o laudo do exame em uma outra janela, com a descrição desse laudo, o nome dos médicos participantes e os revisores do laudo. Havendo alguma imagem disponível para visualização, o usuário pode vê-la em outra janela ativando o botão de visualização de imagem, que fica visível no canto inferior na janela de laudo. Este botão inicia o visualizador de imagens DICOM, permitindo que o usuário navegue pelas imagens, podendo realizar algumas funções como "zoom", controle de brilho e contraste e visualizar imagens lado a lado. As imagens disponíveis para visualização são ditas "on-line". Quando estas imagens tornamse muito antigas no servidor elas são removidas e gravadas em $\mathrm{CD}$, recebendo o status "off-line" no sistema. Atualmente, o período de armazenamento "on-line" das imagens é de aproximadamente cinco meses. Caso seja necessária a recuperação de alguma imagem "off-line", é possível realizar uma consulta no RIS e obter a identificação do CD que contém as imagens referentes ao exame desejado.

\section{DISCUSSÃO}

O principal motivo que desencadeou o desenvolvimento e a implantação de um RIS junto ao Serviço de Radiodiagnóstico do HCFMRP foi a possibilidade de otimização no processo de disponibilização e consulta de laudos pela rede "ethernet" do hospital.

Antes da implantação do RIS, os laudos eram emitidos de forma manual em um formulário que acompanhava o pedido de exame, conferidos pelo especialista, encaminhados para digitação, impressos, corrigidos, reimpressos, assinados e encaminhados ao SAM. Em média, existia um tempo de espera de dois a três dias nesse processo, o que fazia com que a procura por laudos ainda não disponíveis no SAM fosse bastante alta junto ao Serviço de Radiodiagnóstico, contribuindo ainda mais para aumentar o tempo de espera na impressão e encaminhamento dos laudos definitivos. Com a implantação do RIS, esse tempo de espera foi bastante minimizado, pois a partir do momento que o laudo é revisado, ele já está disponível para consulta "on-line". 
Além disso, o laudo definitivo passou a ser impresso diretamente no SAM, utilizando assinatura eletrônica, o que agilizou o processo de distribuição destas informações nos prontuários dos pacientes.

Embora a consulta de laudos tenha sido o principal desencadeador da implantação do RIS, o seu potencial no auxílio ao gerenciamento e controle de qualidade do processo de geração de exames é extremamente grande. Como é armazenado um registro de toda operação realizada no ambiente do sistema, é possível a recuperação de informações estatísticas referentes aos exames realizados e as eventuais perdas e repetições ocorridas, o que pode auxiliar na detecção de pontos críticos (tanto gerenciais quanto técnicos) dentro do fluxo de trabalho do Serviço.

No que se refere ao PACS, a estruturação de um ambiente de radiologia sem filme junto ao Serviço de Radiodiagnóstico do HCFMRP tem sido um dos objetivos do CCIFM/FMRP. Por meio da parceria estabelecida com o CIA do HCFMRP, foi possível a implantação de um servidor DICOM e de uma aplicação "web" para vinculação e disponibilização de imagens e laudos associados. Inicialmente foram disponibilizadas somente imagens de exames de ressonância magnética, mas a estrutura desenvolvida é genérica e deverá ser estendida para as demais modalidades. Também está sendo estudada uma nova versão melhorada do servidor DICOM para gravação das imagens "off-line" em CD e DVD, com maior integração com a base RIS. Na estrutura atual, a inserção do número de acesso do exame no cabeçalho das imagens é feita manualmente pelo técnico, assim como o envio das imagens para o servidor. Porém, já está em andamento junto ao CIA-HCFMRP o desenvolvimento de uma nova versão para o RIS, a qual irá abranger o agendamento de exames e a geração de "worklists", bem como o envio dos dados de forma automatizada para os equipamentos e das imagens para o servidor. Outro aspecto importante na nova versão do RIS será a implantação de modelos estruturados para os laudos, baseados no Index para Diagnóstico Radiológico do Colégio Americano de Radiologia ${ }^{(5)}$.

Como parte do projeto de estruturação do ambiente sem filme, está em andamen- to, também, o desenvolvimento de um sistema para laudos em monitores, de forma a possibilitar o trabalho dos radiologistas sem a necessidade de impressão de filmes (Figura 6). Além das funções básicas de manipulação de imagens, as estações de laudo possibilitarão a seleção de imagenschave para serem vinculadas aos respectivos laudos. Com este novo sistema, as imagens para consulta via "browser" serão as imagens-chave selecionadas pelos ra- diologistas e disponibilizadas em formato jpeg por um servidor "web", ficando o acesso aos arquivos DICOM restrito às estações de laudo. Desta forma, a visualização dos exames laudados vinculados a suas imagens será realizada de maneira mais eficiente exibindo de forma mais direta as imagens de interesse ao exame para o médico que está fazendo a consulta. Também será possível manter um arquivo "on-line" de exames por um período mais

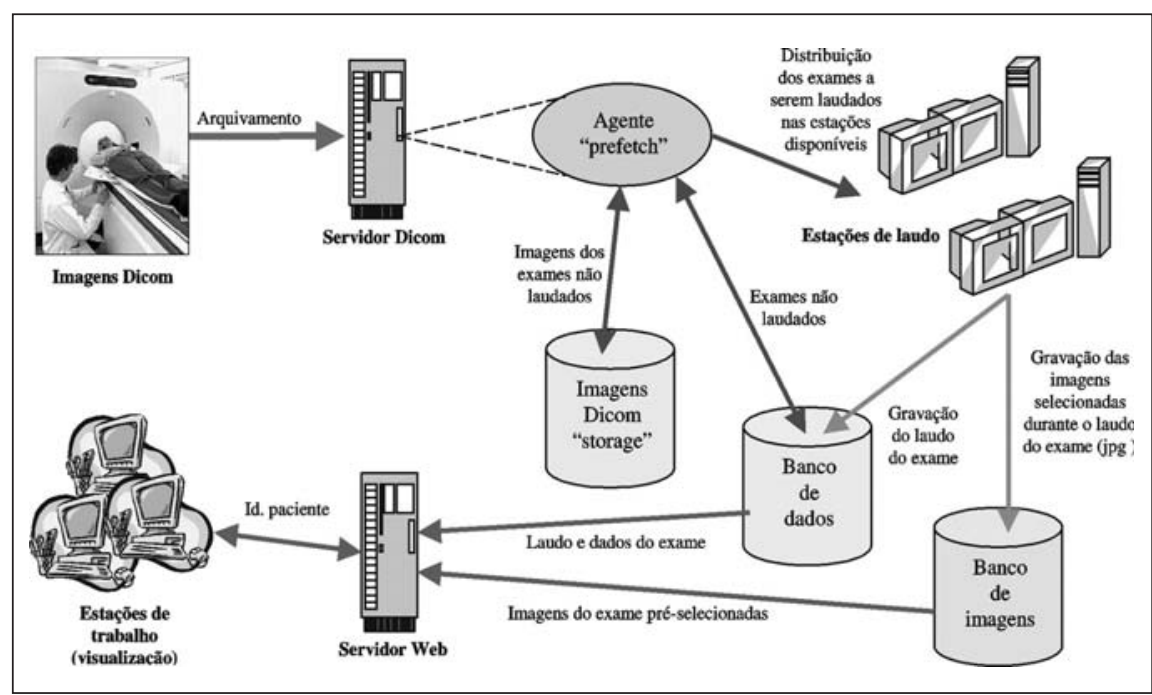

Figura 6. Sistema de laudo eletrônico sem filme ("filmless") com agente de "prefetch" e seleção de imagens durante o laudo do exame.

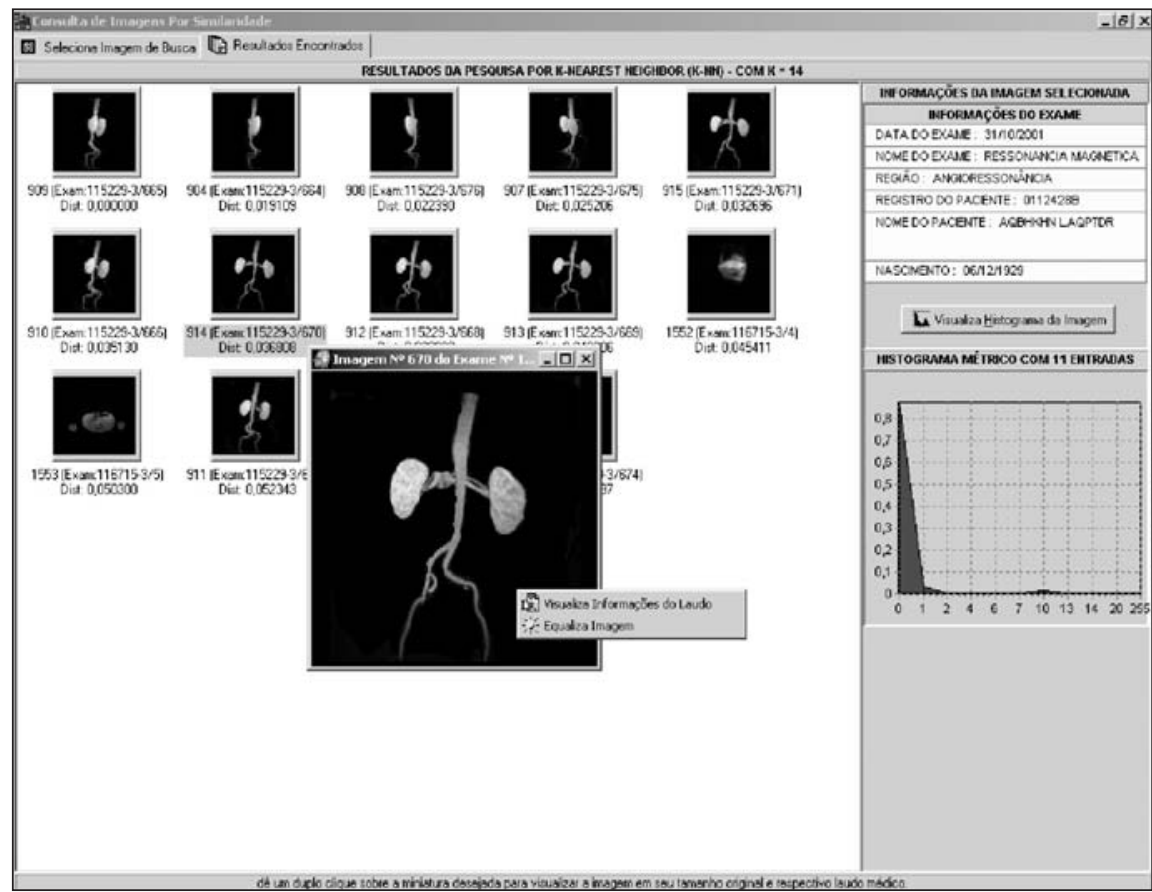

Figura 7. Exemplo de recuperação de imagens médicas por similaridade. 
longo, de três a quatro anos, por exemplo. Atualmente, as imagens DICOM estão disponíveis "on-line" por um período de cinco meses e depois são armazenadas "offline” em CD. Caso os arquivos DICOM sejam necessários por algum motivo específico, poderão ser solicitados junto ao Serviço de Radiodiagnóstico.

Quanto à comunicação entre os equipamentos e o servidor DICOM, observou-se que não houve nenhum problema sistêmico ou falha no padrão para o envio e recebimento das imagens, confirmando a eficácia do padrão DICOM para o gerenciamento e distribuição de imagens dentro do Serviço de Radiodiagnóstico do HCRP. Notou-se também que a rede "ethernet" do hospital atende em grande parte aos requi- sitos para o sistema "filmless", no entanto, há ainda alguns melhoramentos que devem ser feitos para diminuir o tempo de espera para visualização das imagens nas estações da rede "intranet-HCRP”.

Finalmente, está prevista a inclusão, no PACS, de uma função de recuperação de imagens por similaridade de conteúdo visual ("content-based image retrieval" CBIR), por intermédio da extração e comparação automatizadas de atributos pictóricos (Figura 7). As pesquisas para o desenvolvimento do sistema CBIR já foram iniciadas e estão sendo desenvolvidas em parceria com o Grupo de Bases de Dados e Imagens (GBdI) do Instituto de Ciências Matemáticas e de Computação da USP de São $\operatorname{Carlos}^{(6)}$.

\section{REFERÊNCIAS}

1. Passariello R, Venturi G, Campanella V, et al. RIS/ PACS integration in a Web environment. In: Lemke HU, Vannier MW, Inamura K, et al., eds. Berlin: CARS/Springer, 2002:479-84.

2. Digital Imaging and Communication in Medicine, Part 1-10. NEMA Standard Publ. 1992-2000. www.rsna.org

3. Siegel EL. Current state of the art and future trends. In: Siegel EL, Kolodner RM, eds. Filmless radiology. New York, NY: Springer, Health Informatics Series, 1999:3-20.

4. Azevedo Marques PM, Santos AC, Elias Junior J, Goes WM, Castro CR, Trad CS. Implantação de um sistema de informação em radiologia em hospital universitário. Radiol Bras 2000;33:155-60.

5. American College of Radiology. Index for Radiological Diagnosis. 4th ed. Reston, VA: ACR, 1982.

6. Rosa NA, Traina AJM, Traina Junior C. Recuperação de imagens médicas por similaridade em um hospital universitário. Anais do $2 \underline{0}$ Workshop de Informática Médica - WIM'2002. Porto Alegre, RS: Sociedade Brasileira de Computação, 2002. 